\title{
The drawing rules of children: Sequence and direction
}

\author{
ELIZABETH PEMBERTON \\ The University of Kansas, Lawrence, Kansas
}

(Greg B. Simpson, Sponsor)

\begin{abstract}
This study examined the development of some of the directional rules children use in drawing. A broader age range than in previous studies was used to analyze the following drawing rules: top-to-bottom, left-to-right, threading in a continuous clockwise or counterclockwise direction, and nonthreading. These rules were tested across three different age groups (4- and 5-year-olds, 6- and 7-year-olds, and 8- to 10-year-olds) and three figure types (circular, square, and apex). Age and figure type interacted to significantly influence the percentage of rule use. Two tasks, copying and modeling, were compared to test the robustness of these rules to task variation. Task did not greatly influence the selecton of drawing rules. The implications from this study are that the process of drawing is rule-governed, and that some of the rules children use, robust to task variation, shift in kind with age.
\end{abstract}

Many constraints operate on a child who is learning to draw (Freeman, 1972, 1980; Goodnow, 1977). Although a child might hold a certain concept of an object in mind, that concept is not always revealed once the drawing is laid out on paper. Certain performance and task variables can mask a child's true drawing abilities. The goal of this study was to examine some of the rules that operate when a child is drawing. Rule, as used here, is defined as the tendency to proceed in a structured and predictable manner over repeated trials or across time.

The particular drawing rules dealt with here involve the sequence and direction of lines drawn, and whether these lines are "threaded" by using a continuous clockwise or counterclockwise direction from start to finish (Fenson, 1985; Goodnow, 1977). "Nonthreading" implies that the continuous thread was broken: at least one line in the drawing was copied in a direction opposite to that used for the rest of the figure.

The set of figures used in the current study duplicated some used in past investigations of children's drawing rules (Goodnow, 1977; Goodnow \& Levine, 1973; Graham, Berman, \& Ernhart, 1960). Some additional relatively complex figures were also included to determine whether the rules in question applied across a variety of figure types that varied in complexity. To determine whether the same rules of direction would be used by children, regardless of task presentation, copying and modeling tasks were presented. In copying, the child merely

This research was conducted as partial requirement for the doctoral degree in psychology at Pennsylvania State University. Further support for preparation of the manuscript was provided by NICHHD training grant HD07255 to the University of Kansas. I would like to thank Greg Simpson for his sponsorship and comments on an earlier draft. Requests for reprints should be sent to the author, 1043 Indiana St., The University of Kansas, Lawrence, KS 66044. drew the target figure that was placed above his/her paper. In modeling, the child drew the figure after it had been presented in a specific manner by the researcher.

It was predicted that, after an initial period of using nonthreading directional rules or of using inconsistent drawing strategies, children should gradually come to favor clockwise directional rules, and eventually counterclockwise threading rules. Furthermore, specific figuredifferentiated nonthreading rules were expected to eventually replace both clockwise and counterclockwise directional rules as preferred strategies for copying many figures, but not all (e.g., circles). Finally, top-to-bottom and left-to-right rules should both be preferred once schooling starts (6- and 7-year-olds), due partly to the emphasis on reading and writing from left to right and from the top of the page to the bottom.

\section{METHOD}

\section{Subjects}

The 90 right-handed children who participated represented three age groups: 4- and 5-year-olds (mean age $=5 ; 7$ ), 6 - and 7-year-olds (mean age $=6 ; 11$ ), and 8- to 10 -year-olds (mean age $=9 ; 5$ ). Fifteen females and 15 males made up each group. All children tested were from three rural Pennsylvania schools.

\section{Procedure}

The stimuli that were presented for copying were $4 \times 6$ in. laminated cards with stamped designs, the components of which are shown in Figure 1. In addition to copying these shapes, the children copied relatively complex aggregates of some of the shapes. All designs were made using a combination of three rubber stamps: a 1-in. straight line, a $1 / 2$ in. straight line, and a circle $1 / 2$ in. in diameter. Ten different figures were used for the modeling task. These stimuli consisted of small faint marks that indicated to the experimenter where to stamp or draw lines making up figures for the child to copy.

Each child drew on $4 \times 6$ in. white unlined pieces of paper, using a \#2 pencil. Each stimulus card was presented in a clear plastic viewer, which was mounted flat on the table. The child's paper was clamped vertically under the stimulus drawing. 
Copying task. The directions for the copying task, given to each child individually, were as follows: "I'll show you a picture for you to copy. Try to make a drawing that looks just like the one I show you." The copying stimuli were presented in a different scrambled order to each child.

Modeling task: Sequencing. The copying task preceded the modeling task, since information derived from the first task was used by the experimenter in determining the sequence and direction of lines to model in the modeling task. The experimenter always modeled a different order and/or sequence from that used by the child in the copying phase. This was done to test the prediction that the graphic rules children use are robust-that they remain in place even if a model is presented in a manner different from that normally used.

The experimenter used rubber stamps and black ink during the sequencing phase of the modeling task. The directions for this task were as follows: "I'll make a picture with these stamps. When I'm all done, you try to draw the picture with your pencil the same way I did it with these rubber stamps." The order of presentation of stimuli within the modeling task was random.

Modeling task: Direction. In the modeling subtask, direction, the experimenter actually drew the lines to indicate direction. The instructions to the child were as follows: "I'll draw a picture with my pencil. When I'm done, you draw it just the same way I did it with your pencil. Okay?' The stimulus figure was slid into the viewer, where it stayed while the child drew. Five figures were modeled in the sequencing manner; five were modeled to indicate direction.

\section{RESULTS}

\section{Overview}

The following rules were examined: clockwise threading, top-to-bottom, and left-to-right. The dependent measure was the percentage of use of a particular rule when that rule could apply. For example, a score of 40 indicates that the child used a particular rule in $40 \%$ of the instances in which it could apply.

Separate repeated measures analyses of variance (ANOVAs) were performed on the percentage of rule use. Age (4- and 5-year-olds, 6- and 7-year-olds, and 8- to 10-year-olds) and sex were between-subject variables, and figure type (circular, square, and apex) was a withinsubject variable. The data from the copying task were analyzed first alone. Later analyses on a subset of figure types compared tasks, copying and modeling, to determine the effect on rule use of modeling stimuli in a certain way.

Copying task: Clockwise threading. An analysis of the percentage of use of clockwise threading rules in the copying task showed a strong age $\times$ figure type interaction, indicating that certain age groups drew particular figure types differently $[F(4,168)=8.00, p<.001]$. Age and figure type were both main effects [for age, $F(2,84)=3.32, p<.05$; for figure type, $F(2,84)=$ $6.49, p<.01]$. For circular figure types, as subjects' ages increased, the tendency to copy in a clockwise direction steadily decreased (as shown in Table 1). There were U-shaped curves for both square and apex figure types, with more 6- and 7-year-olds using clockwise threading than either the younger or older age groups.

Copying task: Nonthreading. A second analysis of variance was conducted on the percentage of rule use for nonthreading. There was a significant main effect for age $[F(2,84)=3.37, p<.05]$. The 4- and 5-year-olds (mean
Table 1

Mean Percentages of Rules Used for Copying and Modeling Tasks

Drawing Rule Used

\begin{tabular}{|c|c|c|c|c|c|}
\hline & \multicolumn{5}{|c|}{ rawing Rule Used } \\
\hline & \multicolumn{3}{|c|}{ Clockwise Threading } & \multirow[b]{2}{*}{ Top-Bottom } & \multirow[b]{2}{*}{ Left-Right } \\
\hline & Circle & Square & $\overline{\text { Apex }}$ & & \\
\hline \multicolumn{6}{|c|}{ 4- and 5-Year-Olds $(n=30)$} \\
\hline Copying & 50 & 41 & 34 & 84 & 98 \\
\hline Modeling & 46 & 45 & 30 & 85 & 93 \\
\hline \multicolumn{6}{|c|}{ 6- and 7-Year-Olds $(n=30)$} \\
\hline Copying & 16 & 52 & 39 & 97 & 99 \\
\hline Modeling & 13 & 53 & 36 & 93 & 100 \\
\hline \multicolumn{6}{|c|}{8 - to 10 -Year-Olds $(n=30)$} \\
\hline Copying & 10 & 47 & 33 & 94 & 96 \\
\hline Modeling & 9 & 38 & 43 & 94 & 100 \\
\hline
\end{tabular}

percentage of nonthreading rule use $=33 \%$ ) scored significantly higher than the 6- and 7-year-olds (mean percentage rule use $=21 \%$ ). The 8 - to 10 -year-olds (mean $=27 \%$ ) also scored significantly higher than the middle group, as determined by post hoc Tukey tests.

Copying task: Top-to-bottom rule. The three figures to which the top-to-bottom rule could apply can be seen in Figure 1. A repeated measures ANOVA, using age and sex as between-subject variables, was conducted on the percentage of top-to-bottom rule use. The mean percentages of top-to-bottom rule use were high across all age groups; neither age nor sex significantly contributed to these differences in rule use (both $F \mathrm{~s}<1$ ).

Copying task: Left-to-right rule. In a repeated measures ANOVA examining the percentages of use of the rule "draw horizontal lines from left to right," age and sex were again used as between-subject variables. No significant interactions or main effects were found, indicating that neither age nor sex greatly affected the use of this rule [for age, $F(2,84)=1.53, p<.22$; for sex, $F(1,84)=1.49, p<.23]$. Table 1 shows markedly high percentages (all above $90 \%$ ) of left-to-right rule use for all age groups.

Task comparisons. Neither sequence nor direction modeling had a large effect on the drawing rules examined for right-handed children. As Table 1 shows, the percentages of rule use in drawing circular figure types in a clockwise direction were similar for each age group across tasks. Correlations of each child's copying and modeling scores were calculated to help determine the effect of task on the percentage of rule use. A significant positive correlation $[r(88)=.98, p<.0001]$ indicates that most children maintained the threading clockwise rule used in copying circular figure types in the parallel modeling task. Likewise, the percentages of clockwise threading of square $[r(88)=.41, p<.001]$ and apex $[r(88)=.71$, $p<.001]$ figure types were correlated positively and significantly across tasks.

With top-to-bottom rules, scores on the copying task again correlated with scores on the parallel modeling task. 


\section{Threading \\ and Nonthreading}

Circle Square Apex

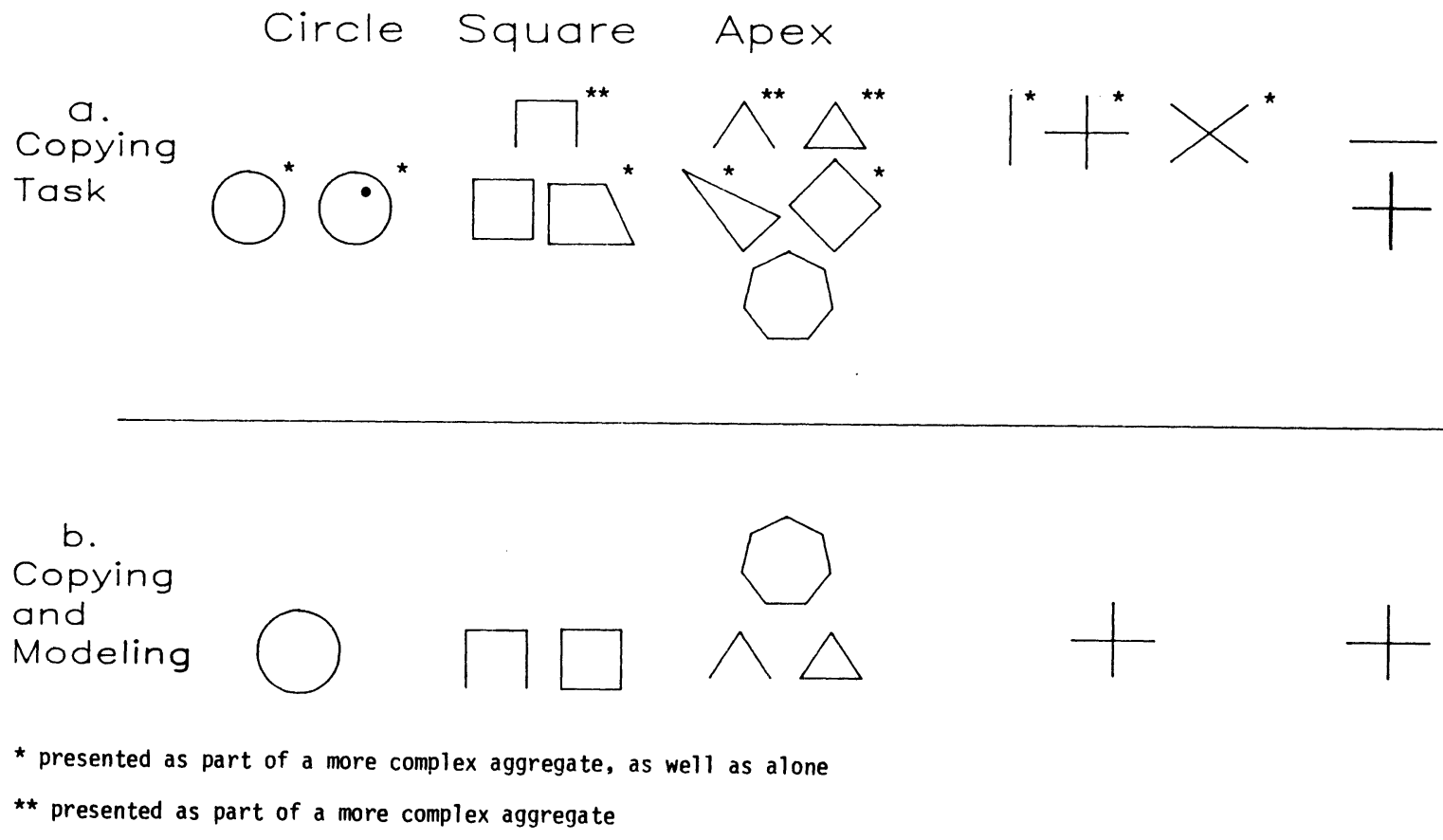

Top-to- Left-
Bottom to-Right 
Goodnow, J. J. (1977). Children drawing. Cambridge, MA: Harvard University Press.

GoodnOw, J. J., \& Levine, R. A. (1973). "The grammar of action": Sequence and syntax in children's copying. Cognitive Psychology, 4, 82-98.

Graham, F. K., Berman, P. W., \& Ernhart, C. B. (1960). Development in preschool children of the ability to copy form. Child Development, 31, 339-359.
Lehman, E. B., \& Goodnow, J. J. (1975). Directionality in copying: Memory, handedness, and alignment effects. Perceptual \& Motor Skills, 41, 863-872.

Pigram, J. S. (1984). The symmetry hypothesis and the perpendicular error: Evidence from discrimination and copying tasks. British Journal of Developmental Psychology, 2, 359-369.

(Manuscript received for publication February 23, 1987.) 